\title{
Comparação das classificações dos nódulos de tireoide ao Doppler colorido descritas por Lagalla e Chammas
}

\author{
Comparation of color Doppler thyroid nodule \\ classifications described by Lagalla and Chammas
}

Marcelo Antonio Serra de Faria', Luiz Augusto Casulari²

\begin{abstract}
RESUMO
Objetivo: Avaliar as classificações da vascularização dos nódulos de tireoide ao Doppler colorido descritas por Lagalla e cols. e Chammas e cols. Métodos: Foram estudados 265 nódulos de tireoide com Doppler colorido e citopatologia. Resultados: No diagnóstico de nódulos com citopatologia maligna, os padrões IV e V de Chammas mostraram sensibilidade de $16,7 \%$, especificidade de $97,6 \%$, valor preditivo positivo de $33,3 \%$, valor preditivo negativo de $94,1 \%$ e acurácia de $92,1 \%$. O padrão III de Lagalla mostrou sensibilidade de $44,4 \%$, especificidade de $19,4 \%$, valor preditivo positivo de $3,9 \%$, valor preditivo negativo de $82,8 \%$ e acurácia de $21,1 \%$. Conclusão: A classificação de Lagalla mostrou baixas sensibilidade e acurácia na detecção de nódulos com citopatologia maligna, enquanto a classificação de Chammas mostrou alta acurácia, porém baixa sensibilidade. O Doppler colorido mostrou-se insuficiente para substituir a punção com agulha fina e o estudo citopatológico no diagnóstico dos nódulos malignos da tireoide. Arq Bras Endocrinol Metab. 2009;53(7):811-7.
\end{abstract}

Descritores

Doenças da glândula tireoide; ultrassonografia Doppler em cores; neoplasias da glândula tireoide; diagnóstico

\section{ABSTRACT}

Objective: Evaluate the vascularization classification of thyroid nodules with color Doppler ultrasonography described by Lagalla and cols. and Chammas and cols. Methods: A total of 265 thyroid nodules were studied with color Doppler and citopathology. Results: In the diagnosis of nodules with malignant citopathology, Chammas's IV and V patterns showed sensibility of $16.7 \%$, specificity of $97.6 \%$, positive predictive value of $33.3 \%$, negative predictive value of $94.1 \%$ and accuracy of $92.1 \%$; Lagalla's III pattern showed sensibility of $44.4 \%$, specificity of $19.4 \%$, positive predictive value of $3.9 \%$, negative predictive value of $82.8 \%$ and accuracy of $21.1 \%$. Conclusion: Lagalla's classification showed low sensibility and accuracy in the detection of nodules with malignant citopathology, while Chammas's classification showed high accuracy, however, low sensibility. Color Doppler was also insufficient to substitute the small needle punction and the citopathologic study in the diagnosis of malignant thyroid nodules. Arq Bras Endocrinol Metab. 2009;53(7):811-7.

Keywords

Thyroid diseases; ultrasonography, Doppler, color; thyroid neoplasms; diagnosis
${ }^{1}$ Centro de Diagnóstico por Imagem Ltda. - Brasília, DF, Brasil ${ }^{2}$ Serviço de Endocrinologia, Hospital Universitário de Brasília (HUB), Brasília, DF, Brasil
Correspondência para:

Marcelo Antonio Serra de Faria Centro Clínico Pacini

SEPS 715/915, Conjunto A, Bloco B, Sala 305

70390-155 - Brasília, DF, Brasil marceloserra.bsb@terra.com.br

Recebido em 15/Mar/2009 Aceito em 12/Jul/2009

\section{INTRODUÇÃO}

$\mathrm{O}$ s nódulos da tireoide são muito comuns na população, sendo encontrados em até $8 \%$ dos adultos pela palpação, em $41 \%$ pela ultrassonografia e em $50 \%$ nas necropsias. Entretanto, apenas $5 \%$ desses nódulos são malignos, representando $1 \%$ de todos os tipos de câncer (1-3). A punção aspirativa com agulha fina (PAAF) é considerada o método de diagnóstico pré-operatório 
mais preciso para a identificação de um nódulo maligno da tireoide $(3,4)$.

O crescente uso da ultrassonografia na avaliação do pescoço levou à detecção de grande número de nódulos impalpáveis. A PAAF de todos os nódulos encontrados teria impacto importante nos sistemas de saúde. Vários estudos tentaram identificar características ultrassonográficas que tivessem boa sensibilidade e especificidade para a diferenciação de nódulos de tireoide benignos e malignos (2-7).

Há controvérsias na literatura sobre a correlação de várias características ultrassonográficas dos nódulos de tireoide com malignidade $(7,8)$. Dentro desse contexto, algumas classificações foram propostas, utilizandose aspectos morfológicos e de vascularização, na tentativa de se estratificar o risco de malignidade e, assim, evitar a realização de biópsias desnecessárias, sem deixar de diagnosticar um número significativo de nódulos malignos (2-11).

Há poucos trabalhos realizados com o Doppler colorido que avaliam as características de vascularização dos nódulos de tireoide e sua associação com malignidade. A classificação mais antiga de nódulos com o uso do Doppler colorido é a de Lagalla e cols. (5), recentemente modificada por Chammas e cols. (3).

Até o momento, as recomendações das sociedades de endocrinologia para indicação de punção de nódulos de tireoide se baseiam principalmente em suas dimensões e consideram as características ultrassonográficas apenas como elementos secundários $(12,13)$.

O objetivo deste trabalho é aferir os resultados das classificações da vascularização dos nódulos de tireoide ao Doppler colorido descritas por Lagalla e cols. (5) e Chammas e cols. (3) na identificação dos nódulos com diagnóstico citopatológico de carcinoma papilar.

\section{MÉTODOS}

Estudo analítico transversal, no qual foram estudados 265 nódulos de tireoide entre novembro de 2005 e julho de 2008, no Centro de Diagnóstico por Imagem Ltda., Brasília (DF).

Os casos foram selecionados retrospectivamente em arquivo, contendo, necessariamente, avaliação dos nódulos com o Doppler colorido, medida do maior eixo destes, idade do paciente na época da punção com agulha fina e relatório citopatológico.

Somente foram incluídos os nódulos com análise citopatológica diagnóstica e com imagens disponíveis em arquivo digital que permitissem a verificação das características ao Doppler colorido descritas nos relatórios dos exames.

O exame ultrassonográfico (modo B) e a avaliação com o Doppler colorido foram realizados em equipamento da marca Toshiba, modelo SSA-550A ( $\mathrm{Ne}$ mio), utilizando-se transdutor linear de alta resolução. As imagens em modo B foram obtidas com frequência central de varredura de $14 \mathrm{MHz}$, um ponto focal, potência de $100 \%$ e faixa dinâmica de $90 \mathrm{~dB}$. Nas imagens com o Doppler colorido, o filtro de parede foi ajustado em $98 \mathrm{~Hz}$ e a frequência de repetição de pulso em 9,4 $\mathrm{KHz}$. Tais ajustes correspondem ao preset instalado no aparelho para estudo da tireoide, tendo sido todos os nódulos estudados com os mesmos parâmetros.

O estudo ultrassonográfico e as punções de todos os nódulos foram realizados por um dos autores (MASF) no Centro de Diagnóstico por Imagem Ltda., Brasília (DF).

O estudo com o Doppler colorido visou a estratificar a vascularização dos nódulos segundo as classificações descritas por Lagalla e cols. (5) e Chammas e cols. (3). Foram revistos todos os relatórios dos exames que incluíam tais informações.

A classificação descrita por Lagalla e cols. (5) separa os nódulos em três tipos de acordo com a vascularização: tipo I (ausência de vascularização), tipo II (vascularização perinodular) e tipo III (vascularização peri e intranodular) (Figura 1).

A classificação proposta por Chammas e cols. (3) separa os nódulos em cinco padrões: padrão I (ausência de vascularização), padrão II (apenas vascularização periférica), padrão III (vascularização periférica maior ou igual à central), padrão IV (vascularização central maior que a periférica) e padrão $\mathrm{V}$ (apenas vascularização central) (Figura 2).

A punção com agulha fina foi realizada por técnica não aspirativa, à mão livre, sempre guiada por ultrassonografia, sem anestesia, com agulha $30 \times 7 \mathrm{~mm}(22 \mathrm{G})$, tendo o número de punções variado em função da qualidade e quantidade do material obtido (em geral, até três passagens).

As análises citopatológicas foram realizadas em diversos laboratórios de citopatologia de Brasília (DF). Utilizaram-se as colorações de Papanicolaou ou hematoxilina-eosina (HE) (14) e os esfregaços foram considerados apropriados para interpretação citopatológica se contivessem, no mínimo, seis agrupamentos celulares. Os relatórios citopatológicos foram reclassificados 

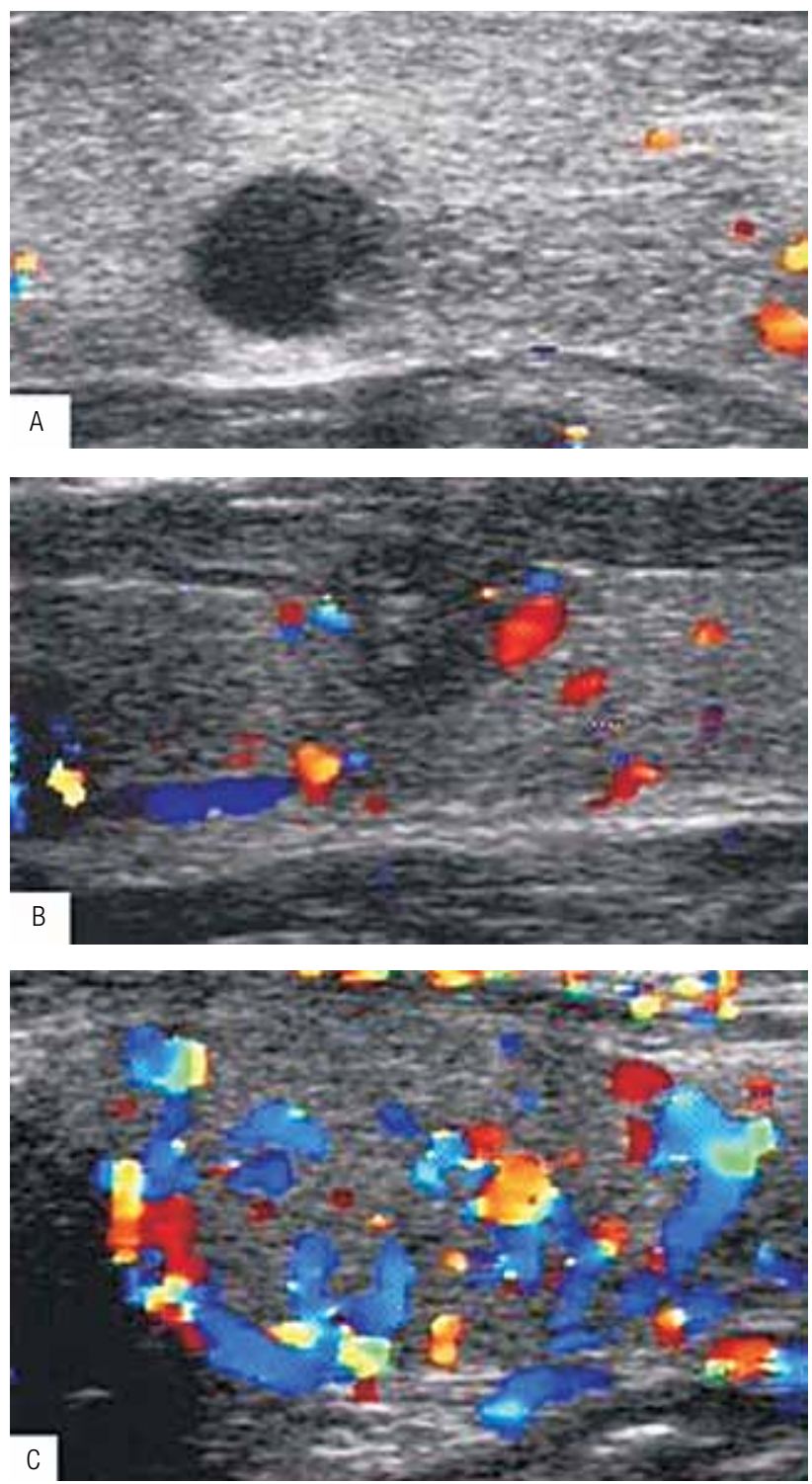

A: padrão I; B: padrão II; C: padrão III.

Figura 1. Classificação da vascularização dos nódulos de tireoide proposta por Lagalla e cols. (5).

de acordo com o sistema recomendado pela Sociedade Papanicolaou de Citologia nos seguintes grupos: benigno, indeterminado, suspeito e maligno (14).

Os dados obtidos foram organizados e analisados por meio dos programas Excel 2007 (Microsoft), Statdisk 10.4.0 (Addison-Wesley, Inc.) e SOCR (Statistics Online Computational Resource - University of California, Los Angeles). Utilizaram-se os testes $t$ de Student $\mathrm{e}$ do qui-quadrado para análise estatística. Considerou-se o nível de significância $\mathrm{p} \leq 0,05$ e os dados são apresentados como média \pm desvio-padrão.

A validade dos testes diagnósticos foi estimada a partir do cálculo da sensibilidade $(\mathrm{a} / \mathrm{a}+\mathrm{c})$, especificidade $(\mathrm{d} / \mathrm{b}+\mathrm{d})$, valor preditivo positivo $(\mathrm{a} / \mathrm{a}+\mathrm{b})$, valor preditivo negativo $(\mathrm{d} / \mathrm{c}+\mathrm{d})$ e acurácia $(\mathrm{a}+\mathrm{d} / \mathrm{a}+\mathrm{b}+\mathrm{c}+\mathrm{d})$, sendo $\mathrm{a}=$ verdadeiros-positivos, $\mathrm{b}=$ falsos-positivos, $\mathrm{c}=$ falsos-negativos e $\mathrm{d}=$ verdadeiros-negativos (15).

O protocolo do estudo foi aprovado pelo Comitê de Ética em Pesquisa da Faculdade de Medicina da Universidade de Brasília, tendo sido dispensado o termo de consentimento livre e esclarecido por se tratar de estudo retrospectivo realizado em arquivo.

\section{RESULTADOS}

Foram estudados 265 nódulos de tireoide, sendo 246 $(92,8 \%)$ em pacientes do sexo feminino e $19(7,2 \%)$ no sexo masculino, e a idade média foi de $50,7 \pm 13,7$ anos, com variação de 17 a 82 anos.

A grande maioria dos nódulos era benigna ou indeterminada, sendo poucos os malignos e suspeitos. Somente se observou diferença significativa $(\mathrm{p}=0,0009)$ entre o tamanho dos nódulos benignos $(20,8 \pm 11,3 \mathrm{~mm}) \mathrm{em}$ relação aos malignos $(14,3 \pm 6,4 \mathrm{~mm})$. Não houve diferença significativa de idade entre os pacientes com nódulos benignos, indeterminados, malignos ou suspeitos.

Nas tabelas 1 a 4, é apresentada a associação entre o resultado citopatológico dos nódulos e o padrão exibido ao Doppler colorido nas classificações de Lagalla e cols. (5) e Chammas e cols. (3).

Analisando-se a associação entre os padrões IV e V de Chammas e cols. (3) e os nódulos com citopatologia maligna, verificou-se sensibilidade de $16,7 \%$, especificidade de $97,6 \%$, valor preditivo positivo de $33,3 \%$, valor preditivo negativo de $94,1 \%$ e acurácia de $92,1 \%$. Na associação entre o padrão III de Lagalla e cols. (5) e os nódulos com citopatologia maligna, verificaram-se sensibilidade de $44,4 \%$, especificidade de $19,4 \%$, valor preditivo positivo de $3,9 \%$, valor preditivo negativo de $82,8 \%$ e acurácia de $21,1 \%$.

Nódulos com padrão de vascularização IV ou V de Chammas e cols. (3) tiveram maior proporção de malignidade $(3[33,3 \%]$ de 9$)$ do que os com padrão I, II ou III $(15[5,9 \%]$ de $256 ; p=0,001$, teste do quiquadrado). Ainda, um nódulo com padrão de vascularização IV ou V de Chammas e cols. (3) exibe chance de malignidade de 8,03 em relação a outro com padrão I, II ou III (odds ratio).

\section{DISCUSSÃO}

A vascularização dos nódulos de tireoide ao Doppler colorido tem sido estudada com classificações distintas $(3,5,7,16,17)$, 

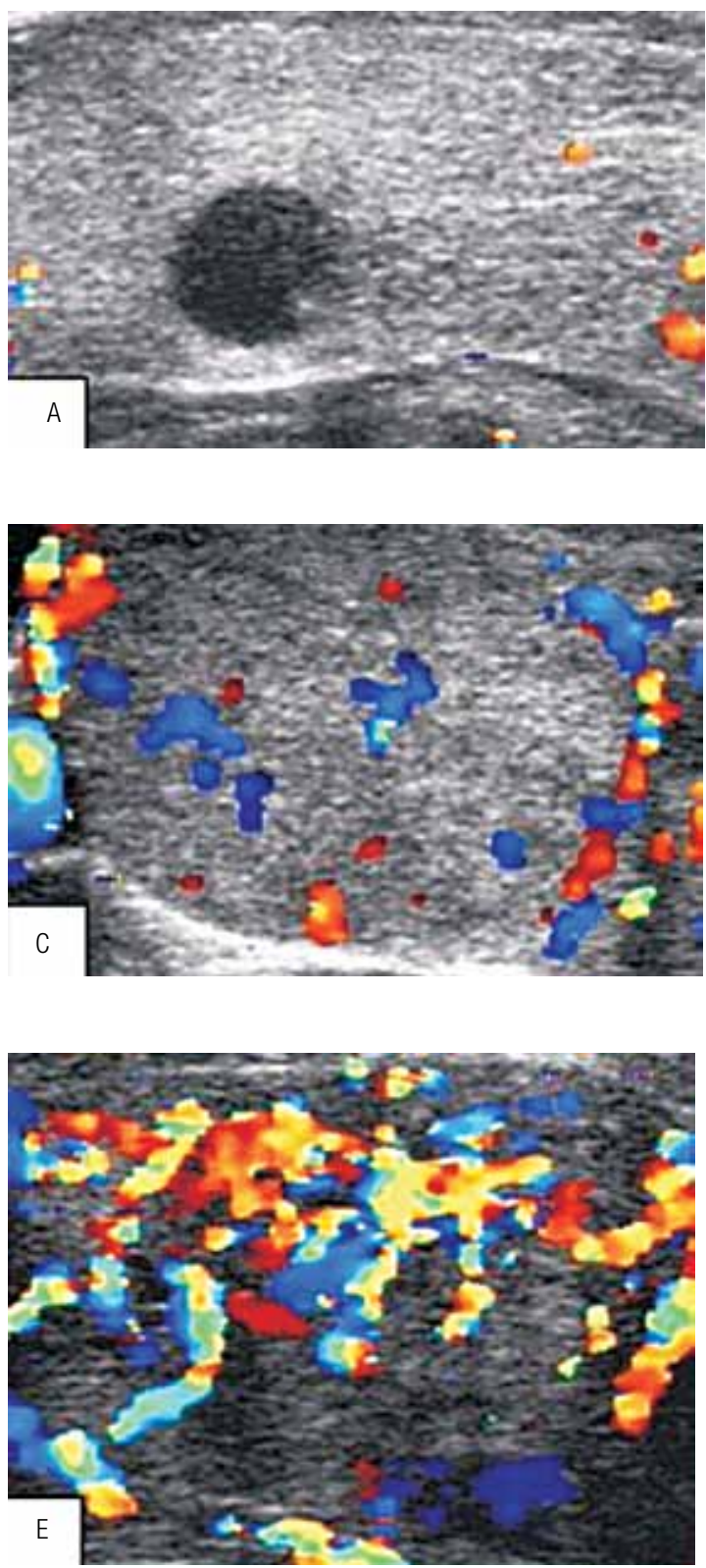

A: padrão I; B: padrão II; C: padrão III; D: padrão III; E: padrão IV; F: padrão V.
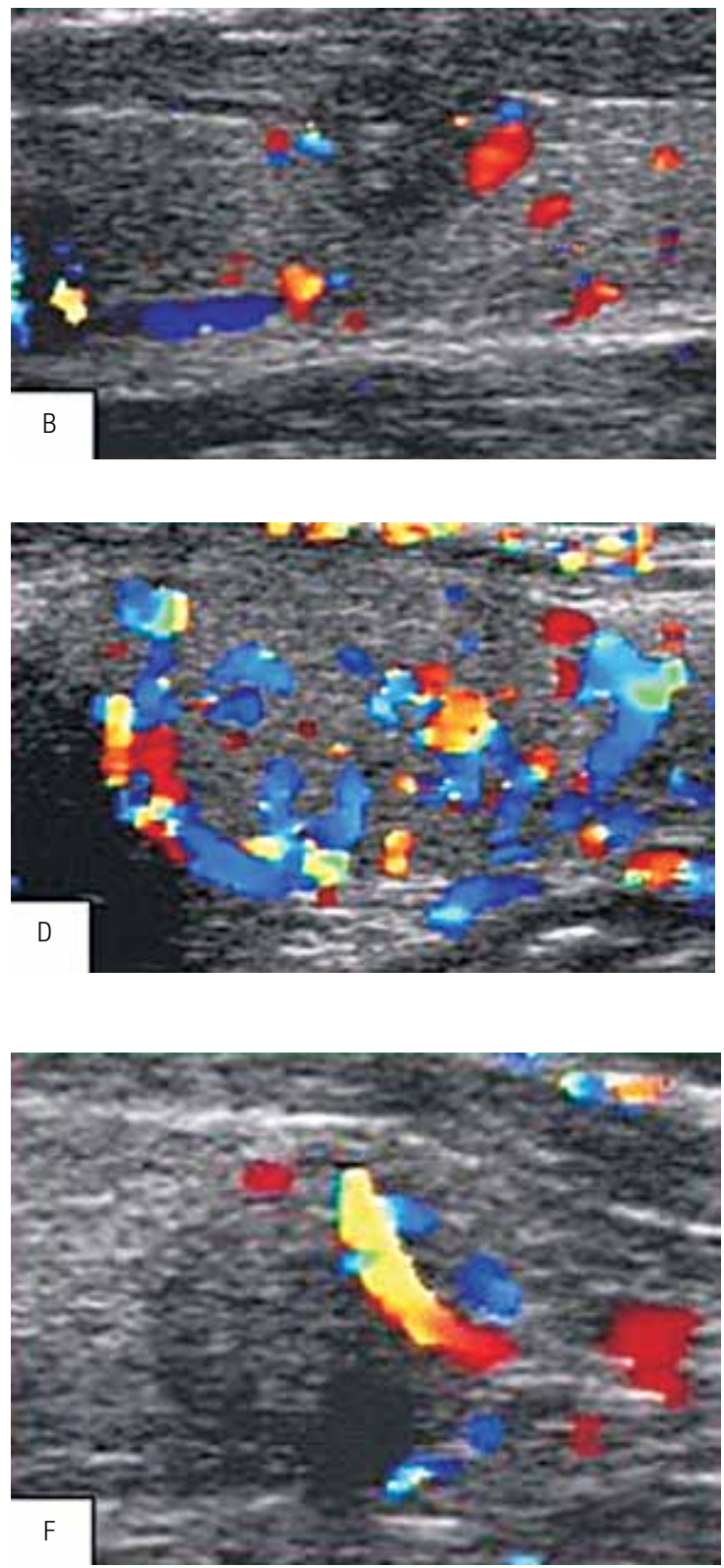

Figura 2. Classificação da vascularização dos nódulos de tireoide proposta por Chammas e cols. (3).

o que dificulta a análise comparativa dos resultados. No presente trabalho, foram avaliadas duas das classificações previamente descritas $(3,5)$, que utilizam apenas o Doppler colorido, não tendo sido analisado o padrão de fluxo ao Doppler pulsado (índice de resistência).

Foram estudados nódulos de tireoide presentes em pacientes majoritariamente do sexo feminino $(92,8 \%)$ e não se observaram diferenças significativas de idade entre os grupos de pacientes com nódulos benignos e malignos.

Neste estudo, foi utilizada a técnica não aspirativa de punção de nódulos da tireoide, que tem resultados similares aos da aspirativa (18), usada por Chammas e cols. (3), enquanto Lagalla e cols. (5) não fornecem tal informação.

No presente estudo, considerou-se como referencial principal de análise o resultado de citopatologia maligna, formato utilizado na maioria dos estudos existentes na literatura, o que se justifica pela excelente acurácia no diagnóstico do carcinoma papilar da tireoide (3,7$9,19)$. Também foi verificado que a proporção de citopatologias malignas $(6,8 \%)$ foi semelhante à de estudos similares $(3,5,8,9)$. 
Tabela 1. Distribuição dos nódulos benignos $(n=149)$ de acordo com as classificações de Lagalla e cols. (5) e Chammas e cols. (3)

\begin{tabular}{ccc}
\hline Padrão & $\begin{array}{c}\text { Lagalla e cols. } \\
\mathbf{n}(\%)\end{array}$ & $\begin{array}{c}\text { Chammas e cols. } \\
\mathbf{n}(\%)\end{array}$ \\
\hline I & $0(0)$ & $0(0)$ \\
II & $27(18,1)$ & $27(18,1)$ \\
III & $122(81,9)$ & $121(81,2)$ \\
IV & - & $1(0,7)$ \\
V & - & $0(0)$ \\
Total & $149(100,0)$ & $149(100,0)$ \\
\hline
\end{tabular}

Tabela 2. Distribuição dos nódulos indeterminados $(n=93)$ de acordo com as classificações de Lagalla e cols. (5) e Chammas e cols. (3)

\begin{tabular}{ccc}
\hline Padrão & $\begin{array}{c}\text { Lagalla e cols. } \\
\mathbf{n} \text { (\%) }\end{array}$ & $\begin{array}{c}\text { Chammas e cols. } \\
\mathbf{n}(\%)\end{array}$ \\
\hline I & $1(1,1)$ & $1(1,1)$ \\
II & $18(19,3)$ & $18(19,3)$ \\
III & $74(79,6)$ & $69(74,2)$ \\
IV & - & $5(5,4)$ \\
V & - & $0(0)$ \\
Total & $93(100,0 \%)$ & $93(100,0 \%)$ \\
\hline
\end{tabular}

Tabela 3. Distribuição dos nódulos suspeitos $(n=5)$ de acordo com as classificações de Lagalla e cols. (5) e Chammas e cols. (3)

\begin{tabular}{ccc}
\hline Padrão & $\begin{array}{c}\text { Lagalla e cols. } \\
\mathbf{n}(\%)\end{array}$ & $\begin{array}{c}\text { Chammas e cols. } \\
\mathbf{n}(\%)\end{array}$ \\
\hline I & $0(0)$ & $0(0)$ \\
II & $2(40)$ & $2(40)$ \\
III & $3(60)$ & $3(60)$ \\
IV & - & $0(0)$ \\
V & - & $0(0)$ \\
Total & $5(100,0)$ & $5(100,0)$ \\
\hline
\end{tabular}

Tabela 4. Distribuição dos nódulos malignos $(n=18)$ de acordo com as classificações de Lagalla e cols. (5) e Chammas e cols. (3)

\begin{tabular}{ccc}
\hline Padrão & $\begin{array}{c}\text { Lagalla e cols. } \\
\mathbf{n} \text { (\%) }\end{array}$ & $\begin{array}{c}\text { Chammas e cols. } \\
\mathbf{n} \text { (\%) }\end{array}$ \\
\hline I & $0(0)$ & $0(0)$ \\
II & $10(55,6)$ & $10(55,6)$ \\
III & $8(44,4)$ & $5(27,8)$ \\
IV & - & $2(11,1)$ \\
V & - & $1(5,5)$ \\
Total & $18(100,0)$ & $18(100,0)$ \\
\hline
\end{tabular}

Os nódulos benignos puncionados foram maiores do que os malignos $(20,8 \mathrm{~mm} \times 14,3 \mathrm{~mm} / \mathrm{p}=0,0009$, teste $t$ de Student), o que difere de outros estudos que não apontaram diferenças $(12,20)$ ou mostraram o inverso $(3)$.
Esses resultados díspares poderiam ser explicados por diferentes critérios de seleção dos nódulos avaliados.

Como o exame de referência para o diagnóstico préoperatório do carcinoma papilar é a punção com agulha fina, a baixa sensibilidade do Doppler colorido no diagnóstico de malignidade em nódulos de tireoide tornaria seu papel secundário na propedêutica diagnóstica.

É frequente, na literatura, o relato de nódulos malignos pouco vascularizados, bem como de nódulos benignos hipervascularizados $(8,16,19,21,22)$. Embora, neste estudo, tenha sido demonstrado risco maior de malignidade na presença de vascularização central predominante nos nódulos de tireoide, a ausência de fluxo central não indica com segurança benignidade, pois uma proporção significativa de carcinomas papilares apresentou tal característica (Figuras 3 e 4).

Chammas e cols. (3) demonstraram vascularização interna em 100 dos 177 nódulos estudados $(56,5 \%)$ e, no presente estudo, foi verificada vascularização interna em 207 dos 265 nódulos $(78,1 \%)$. Como a proporção de carcinomas papilares com fluxo interno encontrada neste estudo $(55,6 \%)$ foi menor do que no citado trabalho $(84,6 \%)$, é provável que as populações estudadas exibam proporções diferentes de carcinomas papilares pouco vascularizados. Uma das possíveis explicações para tal diferença seria o tamanho médio dos nódulos malignos encontrados $(14,3 \mathrm{~mm})$, menor do que no estudo original de Chammas (23) (38,8 mm).

Verificou-se dificuldade na distinção entre os nódulos com vascularização central semelhante à periférica $\mathrm{e}$ os com leve predomínio da central, o que poderia possibilitar interpretações distintas dos padrões descritos por Chammas e cols. (3) por diferentes observadores. Tal viés poderia ser minimizado com o desenvolvimento de critérios menos subjetivos, incorporando-se novas tecnologias na avaliação computadorizada das imagens adquiridas. Diferenças na sensibilidade do Doppler nos equipamentos de ultrassonografia utilizados nos diversos estudos recentes não teriam interferência significativa na classificação dos nódulos mais bem vascularizados. Entretanto, a ultrassonografia é um método essencialmente operador dependente. Portanto, uma descrição menos rígida, apenas mencionando a presença de significativa vascularização central, poderia ser mais segura e, inclusive, foi utilizada em outros estudos $(7,20)$.

Quanto à classificação proposta por Lagalla e cols. (5), sua utilidade prática atual é reduzida, pois não é discriminada a quantidade de fluxo intranodular. A proposta original de maior risco de malignidade em nó- 


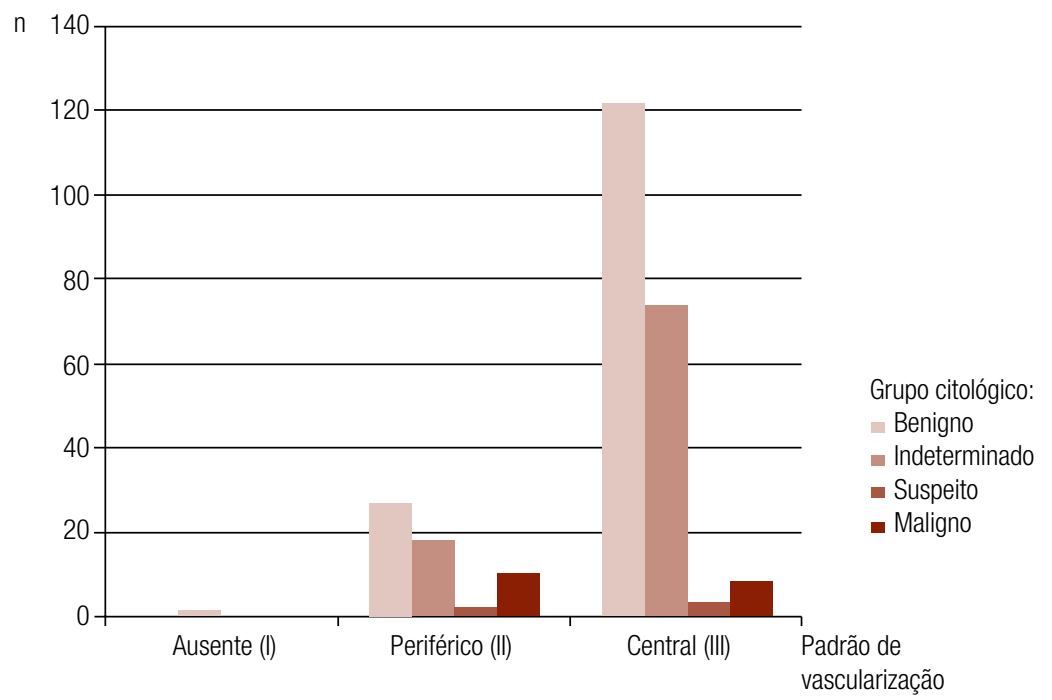

Figura 3. Distribuição dos nódulos de acordo com o padrão de vascularização de Lagalla e cols. (5) e o grupo citológico.

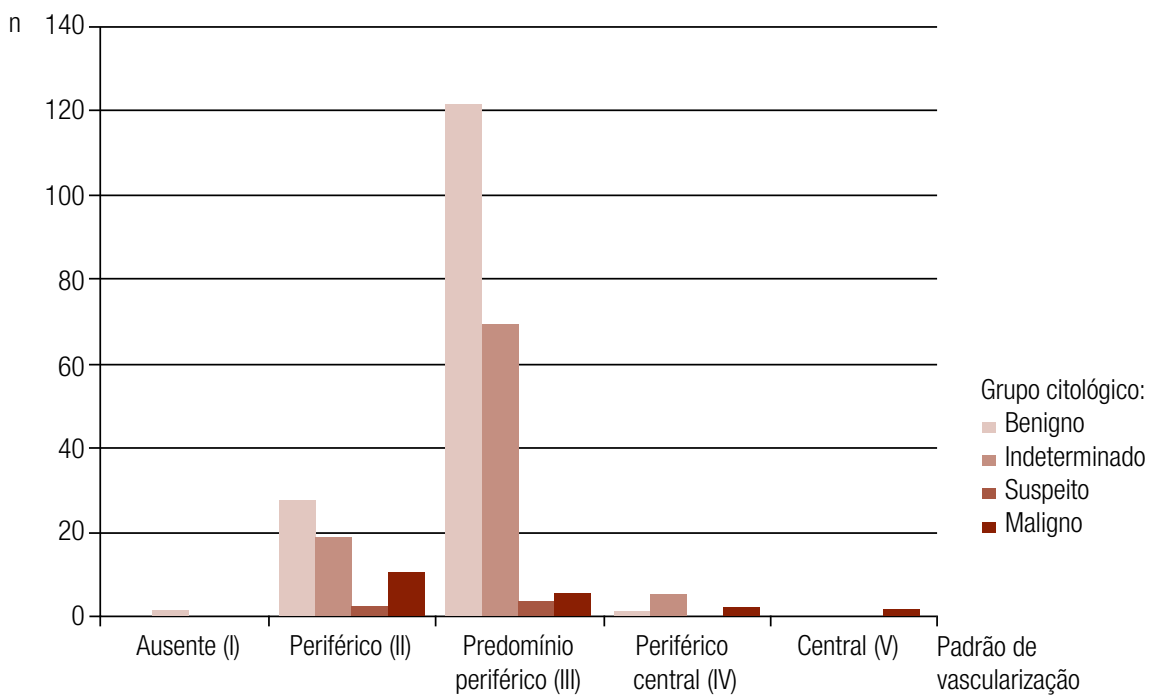

Figura 4. Distribuição dos nódulos de acordo com o padrão de vascularização de Chammas e cols. (3) e o grupo citológico.

dulos com vascularização interna (padrão III) não foi verificada por este e por outros estudos $(3,24)$.

Nossos resultados e a revisão da literatura vão ao encontro dos consensos das sociedades de endocrinologia $(13,25)$ que consideram o tamanho $(>1 \mathrm{~cm})$ como o principal fator na escolha dos nódulos de tireoide que deveriam ser puncionados. Acredita-se que as principais características suspeitas à ultrassonografia (hipoecogenicidade e presença de microcalcificações) (8) e a presença de significativo fluxo intranodular ao Doppler colorido sejam elementos secundários e auxiliares na seleção de nódulos menores ou presentes em tireoides multinodulares que tenham maior probabilidade de serem malignos.

Existem poucos estudos na literatura avaliando a utilização do Doppler pulsado, com medida do índice de resistência arterial, nos nódulos da tireoide, alguns com bons resultados (3,26-28), principalmente na tentativa de diferenciação das neoplasias foliculares malignas no grupo citológico indeterminado. Outros estudos não encontraram utilidade para tal recurso $(29,30)$. Essa técnica não é sequer citada nos consensos especializados $(13,25)$ e, por ser de execução mais difícil, demorada e de reprodutibilidade questionável, acredita-se que es- 
tudos futuros ainda são necessários antes que possa ser incorporada à prática clínica.

Em conclusão, a classificação descrita por Lagalla e cols. (5) mostrou baixas sensibilidade e acurácia na detecção de nódulos com citopatologia maligna e, portanto, não deveria mais ser utilizada. Por outro lado, a classificação descrita por Chammas e cols. (3) apresentou alta acurácia, mas também baixa sensibilidade na detecção dos nódulos com citopatologia maligna. Assim, o Doppler colorido não deve substituir a punção com agulha fina e o estudo citopatológico no diagnóstico dos nódulos malignos da tireoide, mas pode auxiliar na escolha dos nódulos que devem ser biopsiados.

Declaração: os autores declaram não haver conflitos de interesse científico neste estudo.

\section{REFERÊNCIAS}

1. Mortensen JD, Woolner LB, Bennett WA. Gross and microscopic findings in clinically normal thyroid glands. J Clin Endocrinol Metab. 1955;15(10):1270-80.

2. Reading CC, Charboneau JW, Hay ID, Sebo TJ. Sonography of thyroid nodules: a "classic pattern" diagnostic approach. Ultrasound Q. 2005;21(3)157-65.

3. Chammas MC, Gerhard R, Oliveira IRS, Widman A, Barros N, Durazzo $M$, et al. Thyroid nodules: evaluation with power Doppler and duplex Doppler ultrasound. Otolaryngol Head Neck Surg. 2005;132(6):874-82.

4. Rausch P, Nowels K, Jeffrey RB. Ultrasonographically guided thyroid biopsy: a review with emphasis on technique. J Ultrasound Med. 2001;20(1):79-85.

5. Lagalla R, Caruso G, Romano M, Midiri M, Novara V, Zappasodi F. Eco-color-Doppler nella patologia tiroidea. Radiol Med. 1993;85(5 Suppl 1):109-13.

6. YokozawaT, Fukata S, Kuma K, Matsuzuka F, Kobayashi A, Hirai K, et al. Thyroid cancer detected by ultrasound-guided fine needle aspiration biopsy. World J Surg. 1996;20(7):848-53.

7. Frates $M$, Benson C, Doubilet $P$, Cibas ES, Marqusee E. Can color Doppler sonography aid in the prediction of malignancy of thyroid nodules? J Ultrasound Med. 2003;22(2):127-31.

8. Iannuccilli JD, Cronan JJ, Monchik JM. Risk for malignancy of thyroid nodules as assessed by sonographic criteria: the need for biopsy. J Ultrasound Med. 2004;23(11):1455-64.

9. Papini E, Guglielmi R, Bianchini A, Crescenzi A, Taccogna S, Nardi $F$, et al. Risk of malignancy in nonpalpable thyroid nodules: predictive value of ultrasound and color Doppler features. J Clin Endocrinol Metab. 2002;87(5):1941-6.

10. Wienke JR, Chong WK, Fielding JR, Zou KH, Mittelstaedt CA. Sonographic features of benign thyroid nodules: interobserver reliability and overlap with malignancy. $\mathrm{J}$ Ultrasound Med. 2003;22(10):1027-31.

11. Chan BK, DesserTS, McDougall IR, Weigel RJ, Jeffrey Jr. RB. Common and uncommon sonographic features of papillary thyroid carcinoma. J Ultrasound Med. 2003;22(10):1083-90.

12. Kim EK, Park CS, Chung WY, Oh KK, Kim DI, Lee JT, et al. New sonographic criteria for recommending fine-needle aspiration biopsy of nonpalpable solid nodules of the thyroid. AJR. 2002;178(3):687-91.
13. Cooper DS, Doherty GM, Haugen BR, Kloos RT, Lee SL, Mandel $\mathrm{SJ}$, et al. Management guidelines for patients with thyroid nodules and differentiated thyroid cancer:The American Thyroid Association Guidelines Taskforce. Thyroid. 2006;16(2):1-33.

14. The Papanicolaou Society of Cytopathology Task Forces on Standards of Practice. Guidelines of the Papanicolaou Society of Cytopathology for the examination of fine-needle aspiration specimens from thyroid nodules. Diagn Cytopathol. 1996;15(1):84-9.

15. Pereira MG. Epidemiologia: teoria e prática. 1. ed. 6. reimp. Rio de Janeiro: Guanabara Koogan; 1995.

16. Rago T, Vitti P, Chiovato L, Mazzeo S, De Liperi A, Miccoli P, et al. Role of conventional ultrasonography and color flow-Doppler sonography in predicting malignancy in cold thyroid nodules. Eur $\mathrm{J}$ Endocrinol. 1998;138(1):41-6.

17. Cerbone G, Spiezia S, Colao A, Di Sarno A, Assanti AP, Lucci R, et al. Power Doppler improves the diagnostic accuracy of color Doppler ultrasonography in cold thyroid nodules: follow-up results. Horm Res. 1999;52(1):19-24.

18. Tublin ME, Martin JA, Rollin LJ, Pealer K, Kurs-Lasky M, Ohori NP. Ultrasound-guided fine-needle aspiration versus fine-needle capillary sampling biopsy of thyroid nodules: does technique matter? J Ultrasound Med. 2007;26(12):1697-701.

19. Stacul F, Bertolotto M, De Gobbis F, Calderan L, Cioffi V, Romano A, et al. US, colour-Doppler US and fine-needle aspiration biopsy in the diagnosis of thyroid nodules. Radiol Med. 2007;112(5):751-62.

20. Lyshchik A, Drozd V, DemidchikY, Reiners C. Diagnosis of thyroid cancer in children: value of gray-scale and power Doppler US. Radiology. 2005;235(2):604-13.

21. Bartolotta TV, Midiri M, Runza G, Galia M, Taibbi A, Damiani L, et al. Incidentally discovered thyroid nodules: incidence, and greyscale and colour Doppler pattern in an adult population screened by real-time compound spatial sonography. Radiol Med. 2006;111(7):989-98.

22. Camargo R, Tomimori E, Knobel M, Medeiros-Neto G. Preoperative assessment of thyroid nodules: role of ultrasonography and fine needle aspiration biopsy followed by cytology. Clinics. 2007:62(4):411-8.

23. Chammas MC. Contribuição do dúplex-Doppler colorido ao estudo dos nódulos da tireoide. [tese de doutorado]. São Paulo (SP): Universidade de São Paulo; 2001. 154 p.

24. Varverakis E, Neonakis E, Tzardi M, Chrysos E. Role of color Doppler ultrasonography in the preoperative management of cold thyroid nodules. Hormones. 2007;6(1):44-51.

25. Maia AL, Ward LS, Carvalho GA, Graf H, Maciel RMB, Maciel LMZ, et al. Nódulos de tireóide e câncer diferenciado de tireóide: consenso brasileiro. Arq Bras Endocrinol Metab. 2007;51(5):867-93.

26. De Nicola H, Szejnfeld J, Logullo AF, Wolosker AMB, Souza LRMF, Chiferi, VJ. Flow pattern and vascular resistive index as predictors of malignancy risk in thyroid follicular neoplasms. J Ultrasound Med. 2005;24(7):897-904.

27. Miyakawa M, Onoda N, Etoh M, Fukuda I,Takano K, OkamotoT, et al. Diagnosis of thyroid follicular carcinoma by the vascular pattern and velocimetric parameters using high resolution pulsed and power Doppler ultrasonography. Endocrine Journal. 2005;52(2):207-12.

28. Bakhshaee M, DavoudiY, Mehrabi M, Layegh P, Mirsadaee S, Rad MP, et al. Vascular pattern and spectral parameters of power Doppler ultrasound as predictors of malignancy risk in thyroid nodules. Laryngoscope. 2008;118(12):2182-6.

29. Holden A. The role of colour and duplex Doppler ultrasound in the assessment of thyroid nodules. Australasian Radiology. 1995;39:343-9.

30. Tamsel S, Demirpolat G, Erdogan M, Nart D, Karadeniz M, Uluer $\mathrm{H}$, et al. Power Doppler US patterns of vascularity and spectral Doppler US parameters in predicting malignancy in thyroid nodules. Clin Radiol. 2007;62(3):245-51. 